\title{
Cuidados paliativos e medicina de família e comunidade: conceitos e interseções
}

\author{
Lilian Hennemann-Krause, ${ }^{1}$ Letícia A. Freitas, ${ }^{2}$ Priscila M. N. Daflon ${ }^{2 *}$
}

\begin{abstract}
Resumo
Apesar dos avanços tecnológicos, muitas doenças não são passíveis de terapia curativa, tornando-se crônicas e progressivas, tendo a morte como seu desfecho irremediável. Estima-se que cerca de 20 milhões de pessoas ainda morrem no mundo sem acesso à atenção que poderia minimizar seu sofrimento e os sintomas de suas doenças, em sua maioria crônicas. Os cuidados paliativos (CP) são uma área em crescimento, porém, o número efetivo de profissionais capacitados para lidar com o paciente sem expectativa de terapia curativa éinsuficiente.Por meio da revisão bibliográfica narrativa dos pilares conceituais da Estratégia de Saúde de Família, da Atenção Primária à Saúde e dos princípios dos $\mathrm{CP}$, o presente artigo tem por objetivo identificar os pontos que fazem a interseção entre estes e a medicina de família e comunidade (MFC), visto que utilizam atributos semelhantes, o que os torna capazes de acompanhar pacientes cujos sintomas específicos das doenças crônicas ou relacionados com o fim da vida demandam uma necessidade de formação profissional diferenciada. Esta formação permite uma melhor abordagem do paciente e de seus familiares em questões relacionadas com terminalidade, controle da dor e outros sintomas, envolvendo o cuidado em muitas esferas (física, social, cultural, psíquica e espiritual) de forma integrada e coordenada com os demais serviços de saúde. A Estratégia de Saúde da Família apresenta maior capacidade de sustentação em termos de abrangência e acompanhamento continuado dessa população, sendo um ator em potencial para garantir esse cuidado à saúde.
\end{abstract}

Descritores: Atenção primária à saúde; Cuidados paliativos; Medicina de família e comunidade; Saúde da família.

\section{Abstract \\ Palliative care and family and community medi- cine: concepts and intersections}

Despite technological advances, many diseases are not amenable to curative therapy, becoming chronic and progressives, having death as his hopeless outcome. It is estimated that about 20 million people still die in the world without access and attention which could minimize his suffering and symptoms of the disease, chronic in the majority of the cases. Palliative Care (PC) is a growing area; however, the actual number of professionals trained to deal with the patient without expectation of curative therapy is insufficient.Through a narrative bibliographical review of the conceptual pillars of the Family Health Strategy, Primary Health Care and the
1. Clínica e Tratamento da Dor. Hospital Universitário Clementino Fraga Filho. Universidade Federal do Rio de Janeiro. Rio de Janeiro, RJ, Brasil.

2. Departamento de Medicina Integral e Familiar. Hospital Universitário Pedro Ernesto. Universidade Estadual do Rio de Janeiro. Rio de Janeiro, RJ, Brasil.

*Endereço para correspondência: Departamento de Medicina Integral e Familiar, HUPE, UERJ. Boulevard Vinte e Oito de Setembro, 77 Rio de Janeiro, RJ, Brasil. CEP: 20550-030.

E-mail: dra.priscilamoutinho@gmail.com

Revista HUPE, Rio de Janeiro, 2016;15(3):286-293 doi: 10.12957/rhupe.2016.30644 Recebido em 26/06/2016. Aprovado em 29/09/2016.

principles of $\mathrm{PC}$, this article aims to identify the points that make the intersection between the $\mathrm{CP}$ and the Family and Community Medicine (MFC), as they use similar attributes, which enable them to monitor patients whose specific symptoms of chronic diseases or those related to the end of life demand a need for differentiated training. This training allows for better management of patients and their families on issues related to the terminal illness, pain control and other symptoms involving care in many spheres (physical, social, cultural, psychological and spiritual) in an integrated and coordinated manner with the other health services. The Health Family Strategy has greater support capacity in terms of scope and continued monitoring of this population is what makes it a potential actor to ensure this health care.

Keywords: Primary health care; Palliative care; Family practice; Family health.

\section{Resumen}

\section{Cuidados paliativos medicina familiar y comuni-} taria: conceptos e intersecciones

A pesar de los avances tecnológicos, muchas enfermedades no son susceptibles de terapia curativa, volviéndose crónicas y progresivas, teniendo como desenlace irremediable la muerte. Se estima que cerca de 20 millones de personas mueren en el mundo sin tener acceso a la atención que podría minimizar su sufrimiento y los síntomas de su enfermedad, en su mayoría crónica. Los Cuidados Paliativos (CP) son un área en crecimiento. Sin embargo, el número efectivo de profesionales capacitados para manejar al paciente sin expectativa de terapia 
curativa es insuficiente. A través de la revisión bibliográfica narrativa de los pilares conceptuales de la Estrategia de Salud Familiar, Atención Primaria de Salud y de los principios de los Cuidados Paliativos, el presente artículo tiene por objetivo identificar los puntos que conforman la intersección entre los CP y la Medicina Familiar y Comunitaria (MFC), ya que éstos utilizan atributos similares, lo que los hace capaces de acompañar a pacientes cuyos síntomas de enfermedades crónicas o relacionados con el fin de vida requieren de una formación profesional diferenciada. Esta formación permite un mejor manejo del paciente y sus familiares en cuestiones

\section{Introdução}

A medicina moderna dispõe de conhecimento e técnica para prevenção, controle e cura de determinadas doenças. Porém, apesar dos avanços tecnológicos, muitas doenças não são passíveis de terapia curativa, tornando-se crônicas e progressivas, tendo a morte como seu desfecho irremediável. Muitas delas, no seu processo terminal, trazem consigo sintomas desagradáveis e sofrimento para o paciente e seus familiares. ${ }^{1}$

O cuidado a pacientes em fase final de vida está cada vez mais presente no cotidiano dos profissionais de saúde, tornando-se frequente em diversos serviços de saúde que não estão aptos a recebê-los ou mantê-los. Dessa forma, torna-se cada vez mais urgente sua reorganização. ${ }^{2,3}$

A expressão "paciente terminal", em alguns países, é usada para o paciente que tem estimativa de tempo de vida inferior a seis meses devido à existência de câncer avançado, progressivo e incurável.É preciso ter cuidado quanto ao uso da palavra "terminal", pois seu sentido é ambíguo, impreciso e pejorativo, deixando na dúvida, se terminal é o paciente ou a doença. O seu uso, muitas vezes, acaba por rotular o paciente de "terminal", levando a uma ideia de desinvestimento médico e de desistência de cuidados por parte de profissionais e familiares. O paciente com doença avançada se encontra no período mais frágil, exigindo um cuidado mais intenso e humanizado, com priorização do manejo dos sintomas e qualidade de vida. Torna-se necessário assumir uma nova postura assistencial, bem como haver investimentos na formação dos profissionais de saúde.,4

O intenso avanço tecnológico da assistência à saúde trouxe também a transformação da morte, sendo a mesma vista como um fracasso aos olhos dos profissionais de saúde. O estudante de medicina é formado para ver a morte como "o maior dos adversários". A visão da morte como o insucesso de um tratamento produz ansieda- relacionadas con la enfermedad terminal, control del dolor y otros síntomas relacionados con el cuidado en muchos ámbitos (físico, social, cultural, psicológico y espiritual) de forma integrada y coordinada con los demás servicios de salud. La Estrategia de Salud de la Familia tiene una mayor capacidad para sustentar su alcance y dar seguimiento continuo a esta población, siendo un actor potencial para garantizar este cuidado de salud.

Palabras clave: Atención primaria de salud; Cuidados paliativos; Medicina familiar y comunitaria; Salud de la familia.

de e cobrança por parte da população e dos próprios médicos. A morte passou de um fenômeno humano antes compartilhado pela família e pela comunidade para um acontecimento técnico próprio dos hospitais, conhecido como "medicalização social". 3,4

Houve mudança na forma como convivemos e lidamos com a morte, com o processo de morrer e com os pacientes moribundos. Antes, as crianças permaneciam em casa e participavam do processo de morte e de forma gradual se preparavam para a mesma, compreendendo que a morte fazia parte da vida e era natural. Esta atitude se opõe à de uma sociedade em que a morte é vista como tabu, e os debates sobre ela são considerados mórbidos, impróprios; as crianças são afastadas da convivência com esta temática na tentativa de garantir equivocadamente uma proteção. ${ }^{5}$

Para o homem ocidental moderno, a morte passou a ser sinônimo de fracasso, impotência e vergonha. Tenta-se vencê-la a qualquer custo e, quando tal êxito não é atingido, a morte é escondida e negada., ${ }^{4,6}$ Atualmente a morte tem sido um processo solitário, mecânico e desumano. 5

Dentre as práticas de cuidados do fim de vida, alguns conceitos são importantes, dentre eles temos a distanásia, eutanásia ou suicídio assistido e os cuidados paliativos (CP).

A distanásia, conhecida também como obstinação terapêutica ou futilidade médica, define-se quando é feito o uso continuado e persistente de medidas que sustentam a vida de pacientes com doenças avançadas, com manutenção dos sistemas vitais biológicos com pouca ou nenhuma utilidade clínica e consequente retardo da morte?

Já a eutanásia, conhecida na antiguidade como "boa morte", teve seu significado modificado no decorrer dos tempos. Lepargneur descreve a 'eutanásia na atualidade' como "o emprego ou abstenção de proce- 


\section{Artigo de revisão}

dimentos que permitem apressar ou provocar o óbito de um doente incurável, a fim de livrá-lo dos extremos sofrimentos que o assaltam"7,8

Hoje a eutanásia é censurável, assemelhando-se ao homicídio no Código Penal Brasileiro em seu artigo 121, e, como tal, passível de punição. Também o Código de Ética Médica diz em seu $6^{\circ}$ princípio fundamental que "o médico guardará absoluto respeito pelo ser humano e atuará sempre em seu benefício. Jamais utilizará seus conhecimentos para causar sofrimento físico ou moral, para o extermínio do ser humano ou para permitir e acobertar tentativa contra sua dignidade e integridade. E afirma que é vedado ao médico "Abreviar a vida do paciente, ainda que a pedido deste ou de seu representante legal". ${ }^{9}$

Segundo a definição da Organização Mundial de Saúde, revista em 2002, "Cuidado Paliativo é uma abordagem que promove a qualidade de vida de pacientes e seus familiares, que enfrentam doenças que ameacem a continuidade da vida, por meio da prevenção e alívio do sofrimento. Requer a identificação precoce, avaliação e tratamento da dor e outros problemas de natureza física, psicossocial e espiritual". Seus princípios são centrados nas atitudes, comunicação e cuidado, sendo uma abordagem apropriada para os pacientes portadores de doenças crônicas e incuráveis. ${ }^{2,10}$

Os CP, como uma disciplina que inclui assistência, começaram a ser organizados há cerca de 40 anos, tendo seu arcabouço teórico na filosofia do moderno movimento hospice - o cuidar de um ser humano que está morrendo, e de sua família, com compaixão e empatia. ${ }^{11}$

As diretivas antecipadas da vontade (advanced directives) ou living wills são instruções deixadas por escrito pelo paciente, ou por seu procurador, que poderá decidir por ele caso esteja impossibilitado de responder pelos seus atos, sobre os cuidados e as opções terapêuticas possíveis. Esse instrumento faz parte de uma série de cuidados que é chamado na literatura como um processo para garantir uma "boa morte". 4,11

O conceito de "boa morte", no contexto dos cuidados no fim da vida, tem sido utilizado quando estão presentes determinadas características, tais como: morte sem dor; morte ocorrendo com os desejos do paciente sendo respeitados. ${ }^{11}$

Com o desenvolvimento das tecnologias, a morte, que ocorria em ambiente domiciliar, passou a ocorrer em casas de saúde e hospitais, principalmente em unidade de terapia intensiva (UTI), que apresenta um ambiente isolado, com janelas fechadas, luz artificial, temperatura constante mantida pelo ar-condicionadoe equipamentos técnicos, e onde os profissionais da saúde realizam procedimentos altamente sofisticados. Os pacientes muitas vezes solitários se sentem impossibilitados de expressar suas emoções, destinados à solidão. Muitos profissionais de saúde não estão preparados para lidar com o processo de morte, agravando mais ainda o sofrimento dos pacientes. ${ }^{11}$

No Brasil, já foram tomadas iniciativas com vistas à possibilidade de se construir uma rede de cuidados no fim da vida no âmbito do Sistema Único de Saúde (SUS), com a implementação dos Centros de Alta Complexidade em Oncologia (CACON), por meio de equipes de CP, inclusive com suporte domiciliar; e a instituição do Programa Nacional de Assistência à Dor e Cuidados Paliativos do SUS. ${ }^{12}$ Observa-se o crescimento da oferta desses serviços ano a ano, sendo a maioria destes ligados a hospitais especializados e de ensino, por meio de internação, atendimento ambulatorial e domiciliar., ${ }^{2,5}$

Ainda são poucos os serviços de CP para atendimento da demanda populacional, mesmo com a ampliação dos CP como modalidade de atenção. Estima-se que cerca de 20 milhões de pessoas ainda morrem no mundo sem acesso à atenção que poderia minimizar seu sofrimento e os sintomas de suas doenças. ${ }^{3}$

No Estado do Rio de Janeiro, levando-se em conta estimativas do Instituto Nacional de Câncer (INCA) para 2014, ${ }^{13}$ excluindo os casos de câncer de pele não melanoma, o número de pacientes que necessitam de CP por ano, desde o diagnóstico, seja em torno de 12.576 na capital e 27.816 em todo o estado. Este número dobra ao considerarmos os familiares cuidadores que deveriam ser incluídos. Ou seja, 25.152 na capital e 55.632 em todo o estado. ${ }^{13}$

Esses dados nos mostram a demanda existente nesse campo de atuação que, portanto, deve ser objeto de atenção dos profissionais da saúde, educadores e gestores. $^{14}$

As políticas de saúde enfrentam um desafio inerente ao contexto atual, no qual estão presentes a alta tecnologia disponível no mercado e a escassez de recursos para a organização dos serviços de saúde que atendam às necessidades básicas da população. ${ }^{12}$

Os modelos bem-estruturados para assistência no fim da vida devem ser implantados para que haja uma maior otimização destes recursos, com redução do tempo de hospitalização e do uso mais racional da tecnologia hospitalar para evitar as situações cotidianas de abandono e de sofrimento, tanto para os pacientes quanto para seus familiares. $^{12}$

No Brasil, os poucos serviços de CP geralmente es- 
tão ligados a hospitais especializados.Porém, em vários países, a Atenção Primária à Saúde (APS) é considerada o melhor nível de assistência para a prestação e coordenação dos CP de seus usuários. ${ }^{2}$

Isso pode ser levado em consideração num país continental como o Brasil, pois existem locais onde não há um CACON estruturado, nem serviços específicos de atendimento domiciliar com enfoque paliativo. Nestes locais a APS pode desempenhar um importante papel no cuidado de pacientes com câncer avançado, mesmo em locais em que aqueles serviços mostram-se disponíveis e atuantes, estabelecendo uma interface com esses serviços na condução dos casos mais avançados no domicílio. ${ }^{12}$

A expressão APS ${ }^{14}$ é utilizada para conceituar um modelo de organização do sistema de saúde cuja intenção primordial é promover, recuperar ou melhorar a saúde, por meio da organização dos serviços. Esta configura-se como espaço da coordenação das respostas às necessidades dos indivíduos, suas famílias e comunidade, como um dos níveis de atenção do sistema de saúde (sejam as unidades básicas de saúde, sejam as equipes de saúde da família) e tem como atributos o primeiro contato, longitudinalidade, integralidade e coordenação do cuidado. ${ }^{15}$

O primeiro contato, considerado a "porta de entrada" para o sistema de saúde, garante a acessibilidade, pelo acesso geográfico e sócio-organizacional, facilitando as pessoas a receberem os cuidados de saúde. ${ }^{15}$

A longitudinalidade garante a continuidade do cuidado em saúde e o estabelecimento de vínculo e coordenação mais eficiente das ações e serviços do sistema, com redução da utilização de serviços especializados e de hospitalizações, maior satisfação dos usuários e diminuição dos custos, vantajoso para pacientes com doenças crônicas e morbidades múltiplas. ${ }^{15}$

A integralidade está associada a maior quantidade e qualidade de ações de prevenção, maior adesão ao tratamento e satisfação da população. ${ }^{15}$

A coordenação do cuidado se dá por meio de uma boa comunicação, informação, articulação e ordenação nos diversos níveis de atenção, permitindo uma corresponsabilização pelo paciente sob a coordenação dos profissionais da APS. ${ }^{15}$

A Estratégia Saúde da Família (ESF) foi instituída no Brasil em 1994, objetivando a reorganização do modelo de assistência à saúde a partir da atenção primária em conformidade com os princípios do SUS. ${ }^{2}$

A ESF, que tem a APS como o local de suas ações e faz dos atributos da APS os seus pilares, desempenha um importante papel na assistência dos pacientes em CP. Mesmo não sendo originalmente desenvolvidos para ações em CP, pode ser reestruturada para incorporar este modelo, assumindo atribuições importantes. ${ }^{12}$

O Núcleo de Apoio à Saúde da Família (NASF), criado pelo Ministério da Saúde em 2008, tem como objetivo apoiar a consolidação da Atenção Básica no Brasil, ampliando as ofertas de saúde na rede de serviços, assim como a resolutividade, a abrangência e o alvo das ações. ${ }^{16}$ O NASF é formado por uma equipe de profissionais de diversas especialidades que atuam de forma integrada com as equipes de saúde da família, permitindo a construção conjunta de projetos terapêuticos, ampliando e qualificando as intervenções. ${ }^{16,17}$

A descentralização do cuidado se tornou de grande valia em um dos principais objetivos do acompanhamento de pessoas em CP: garantir a melhor qualidade de vida possível, sendo reconhecidos na APS os principais atores dessa descentralização. ${ }^{18}$

$\mathrm{O}$ objetivo no presente artigo é identificar quais são os pontos que fazem a interseção entre os CP e a medicina de família e comunidade, visto que esses utilizam os atributos da APS como seus próprios atributos, sendo capaz de atuar e identificar potencialidades no acompanhamento dos pacientes em CP.

\section{Metodologia}

Revisão bibliográfica narrativa dos pilares conceituais da ESF, da APS e dos princípios dos CP. Para realização das buscas foram utilizadas as bases de dados da Biblioteca Virtual Saúde (BVS) e do Google Acadêmico. Os descritores usados foram: atenção primária à saúde (primary health) AND/OR cuidados paliativos (palliative care) $A N D / O R$ medicina de família e comunidade ( $f a-$ mily practice) AND/OR saúde da família (family health).

Foram inicialmente identificados 26 artigos científicos nas bases de dados citadas. Selecionados nas três línguas: inglês, espanhol e português, cujos temas abordassem conceitos, cuidados em saúde e formação profissional vinculados essencialmente aos CP e medicina de família e comunidade.

Após leitura exploratória, foram selecionados 18 artigos que foram avaliados de forma integral e analítica como relevantes à discussão da questão levantada.

\section{Resultados}

O cuidado paliativo é uma abordagem que tem como objetivo a promoção da qualidade de vida do paciente e de seus familiares no momento de enfrentamento de doenças que ameaçam a continuidade 


\section{Artigo de revisão}

da vida. Envolve o tratamento de sintomas e outros problemas psíquicos, sociais, culturais e espirituais, trazendo uma maior complexidade no cuidado, que é inerente à APS.,11

A importância da atenção primária está vinculada ao cuidado poder ser ofertado em casa com conforto ou segurança. No contexto domiciliar, a equipe de APS seria responsável pelo suporte ao paciente e à família, de maneira a garantir o controle dos sintomas e a dignidade no processo de morte. ${ }^{13}$

A equipe técnica da equipe de ESF é composta por médico e enfermeiro que realizam consulta ambulatorial e visita domiciliar. O técnico em enfermagem realiza os cuidados tanto na Clínica da Família quanto em visitas domiciliares. O agente comunitário de saúde, por estar constantemente presente no território e pelo estreito conhecimento que tem de sua comunidade, constitui um importante elo entre estes pacientes e o restante da equipe, identificando necessidades da população adscrita. ${ }^{12}$

Este cuidado aos doentes terminais no contexto da atenção primária e principalmente pelo fato de ocorrer em âmbito domiciliar, acaba garantindo que este cuidado seja humanizado, pois envolve a família tanto nos cuidados como no amparo afetivo ao paciente. O que acaba por reduzir as complicações e os custos decorrentes de longas internações hospitalares. ${ }^{3}$

Nesse sentido, e em consonância com o SUS, a APS orienta-se pelos princípios da coordenação do cuidado, do vínculo e continuidade, da integralidade, da responsabilização, da humanização, da equidade e da participação social. Ainda como marco legal, a Política Nacional de Humanização defende a identificação das necessidades sociais de saúde. Portanto, a aplicação desses princípios e definições demanda incorporação dos CP na atenção primária. ${ }^{14}$

Muitos pacientes manifestam interesse de que a morte ocorra no domicílio ${ }^{2}$ e para isso necessitam de apoio e cuidado. Esta decisão não costuma ser definitiva, podendo mudar nos últimos dias ou nas horas finais de vida. ${ }^{12,19}$ A falta dessa estrutura leva, muitas vezes, a encaminhamentos desnecessários ou equivocados à UTI, gerando um cuidado inadequado ao paciente, à família. ${ }^{20}$

A APS, pela proximidade geográfica, cultural e emocional desses profissionais contribui para que o cuidado ocorra de forma humanizada. ${ }^{2} \mathrm{Na}$ maioria das vezes, atua como primeiro contato no manejo de sintomas físicos e emocionais e suporte para questões relacionadas aos serviços especializados, laudos, enca- minhamentos. ${ }^{2}$ É a responsável pela continuidade do cuidado e integração das condutas nas transições entre ambientes como, por exemplo, a alta hospitalar para residência. $^{20}$

Para que isso ocorra com sucesso, é fundamental que as agendas dos profissionais de APS estejam organizadas e com flexibilidade para realizar consultas ambulatoriais ou visitas domiciliares, já que pela gravidade do quadro haverá muitas demandas solicitadas tanto para esclarecimento de dúvidas quanto para a escuta das angústias dos pacientes e de seus familiares. Quanto mais próximo da morte estiver o paciente, maiores serão as necessidades deste e dos familiares, exigindo consultas mais frequentes. ${ }^{2}$

A permanência do paciente no domicílio não segue um curso linear, repetidas internações hospitalares poderão ocorrer, sendo de grande importância o estabelecimento de parcerias com serviços emergenciais e hospitalares. ${ }^{12}$

A APS pode identificar demandas que ficaram despercebidas pelos serviços de atenção secundária e emergência. Sendo, também, capaz de integrar a ação dos especialistas e adequar as orientações à realidade sociocultural da família. Um dos princípios básicos do SUS é a integralidade da assistência, o que significa considerar a integralidade do sujeito, dos serviços e dos cuidados, que deve incluir necessariamente os cuidados no final da vida.,14

Em CP, o principal objetivo no apoio à família é ajudá-la a cumprir a função cuidadora, a fim de que a participação no processo de perda que vivencia seja concluída da forma mais saudável possível. A APS, pela proximidade, permite o conhecimento da realidade das famílias envolvidas e o vínculo já estabelecido, sendo o nível de assistência à saúde capaz de possibilitar o cuidado nesse lócus. ${ }^{2}$

Com o cuidado domiciliar, emerge a figura do cuidador. Cuidadores costumam conviver com significativo aumento de sua sobrecarga física, emocional, social, material, financeira e existencial, especialmente quando a doença do paciente está com seu curso avançado. $^{12}$

A doença em fase terminal pode provocar nos familiares uma série de reações emocionais, comportamentais, relacionais. Por este motivo, além dos cuidados prestados ao paciente, é necessário que os profissionais de saúde também direcionem seus esforços aos familiares e/ou pessoas relacionadas. ${ }^{4}$

Observar e analisar situações de pré-morte e pré-luto, tanto na pessoa doente como na família, é, em 
si mesmo, um elemento preventivo de grande riqueza para a equipe.

Os familiares merecem um cuidado especial, desde o instante da comunicação do diagnóstico, uma vez que esse momento tem um enorme impacto sobre eles. Isso faz com que, em muitas circunstâncias, suas necessidades psicológicas excedam as do paciente e, dependendo da intensidade das reações emocionais desencadeadas, a ansiedade familiar torna-se um dos aspectos de mais difícil manejo. ${ }^{5}$

Na organização do processo de trabalho da ESF o planejamento das ações em saúde pode ser realizado por meio do projeto terapêutico singular (PTS), inicialmente desenvolvido na área de saúde mental com o objetivo de definir propostas de ações para o cuidado integral da pessoa, com base em diferentes olhares dos profissionais da equipe, sobre os diversos aspectos da vida da pessoa. De tempos em tempos, esse projeto é reavaliado e as metas são redefinidas. Nele fica evidente a riqueza e a valorização dada pelos profissionais sobre a análise social e familiar. A negociação e pactuação das metas devem necessariamente envolver o paciente e seus familiares, o que permite um maior vínculo do paciente com a equipe de saúde e empodera o paciente em seu acompanhamento. ${ }^{14}$

Com a pactuação do cuidado do paciente, pode-se planejar o cuidado que será ofertado a ele. Pode ser discutido se o óbito ocorrerá no domicílio ou em unidade hospitalar, sempre respeitando a vontade do paciente e de seus familiares. A equipe de saúde orienta a respeito da liberação do atestado de óbito que, caso o óbito ocorra em domicílio, poderá ser preenchido pelo médico de família e comunidade do próprio paciente. Pode ser acordado entre médico e familiares que, se por acaso o óbito ocorrer fora do expediente da clínica, a família tenha um contato do médico e pode contactá-lo para a emissão do atestado. Caso o médico identifique que o paciente se encontra em seus últimos dias, o médico poderá emitir um laudo relatando as patologias do paciente para que a família leve caso paciente faleça em um serviço de emergência e a equipe médica da unidade preencha o atestado com as causas corretas.

$O$ atendimento domiciliar significa muito mais do que simplesmente ir até a casa do doente. Significa estar preparado a se tornar membro de um time. Significa muitas vezes ir até a casa do doente, quando aparentemente "não há nada a ser feito" e oferecer suporte a ele e à família, para que esta consiga cuidar do paciente. ${ }^{20}$

A fim de conquistar um cuidado adequado, o profissional deve conhecer e dominar procedimentos e abor- dagens clássicas em cuidados paliativos e integrá-las na base do conhecimento dos profissionais, entendendo como primordial sua implantação na graduação e formação do médico. ${ }^{12}$

\section{Discussão}

Equivocadamente, apesar do seu crescimento em várias partes do Brasil e do mundo, ainda entende-se a APS como os cuidados de "doenças simples" ou "condições de saúde básicas", sendo na realidade justamente o contrário. O médico de família e comunidade, no seu dia a dia, vive realidades complexas, acompanhando além dos pacientes, seus familiares, compreendendo a dinâmica familiar como a relação se dá entre os membros da família, a comunidade e o contexto no qual estão inseridos. Isso torna mais rico o conhecimento que o médico tem do seu paciente.

O cuidado paliativo é uma área de atuação que está crescendo tanto em qualificação de profissionais quanto em pacientes carentes deste cuidado. Entretanto, o número efetivo de profissionais capacitados para lidar com o paciente sem expectativa de terapia curativa é insuficiente em relação à demanda que vem surgindo.

Hoje, no Brasil, a grande maioria dos pacientes em fim de vida são cuidados pelas equipes de saúde da família, visto que a ESF é o principal e, em muitos casos, o único acesso que o paciente tem ao serviço de saúde.

Pacientes em CP apresentam elevado número de internações e ocupam leitos em enfermarias diversas, distribuídos de forma errática, sem garantia de um cuidado específico ou uma abordagem completa. Por vezes vagam por emergências nas quais são avaliados, e os sintomas são controlados temporariamente, sem a garantia de que os cuidados serão realizados também no domicílio. São levados por familiares para falecerem no hospital, por receio de que o óbito ocorra em casa, muitas vezes contrariando a vontade do paciente. Acabam morrendo sozinhos e distantes física e espiritualmente de seus entes, o que pode vir a complicar o luto dos familiares.

Como visto anteriormente, o médico de família e comunidade abrange o indivíduo por completo. Umaassistência centrada na pessoa do paciente envolve o cuidado com atenção às suas condições físicas, sociais, culturais, psíquicas e espirituais de forma integrada e coordenada com os demais serviços de saúde, sendo um importante ator no acompanhamento e cuidado à saúde. Permite fazer a avaliação de comorbidades, das condições de saúde que podem vir a necessitar de internação e, em contato, para que ao retornar para 


\section{Artigo de revisão}

casa mantenham-se os cuidados iniciados, tais como: a realização de ajuste da medicação para a dor, controle dos sintomas e, também, alívio de um sofrimento como o medo da morte.

A acessibilidade à APS facilita para que esta abordagem traga maior conforto e redução do sofrimento do paciente e seus familiares, permitindo que esse passe os seus últimos dias de vida da forma mais digna possível.

\section{Conclusão}

Com o aumento da demanda de doenças crônicas, surge a necessidade do cuidado abrangente e especializado para a manutenção da saúde e garantia da qualidade de vida da população.

Os sintomas específicos das doenças crônicas ou relacionadas com o fim da vida demandam uma formação profissional que permita uma melhor abordagem do paciente e seus familiares para questões relacionadas com a terminalidade, controle da dor e outros sintomas, envolvendo o cuidado em muitas esferas (física, social, cultural, psíquica e espiritual) de forma integrada e coordenada com os demais serviços de saúde.

Os pontos de interseção entre os $\mathrm{CP}$ e a medicina de família e comunidade são identificados em vários aspectos, como abordagem biopsicossocial e espiritual, cuidado domiciliar, longitudinalidade e coordenação do cuidado.

Por meio da abordagem biopsicossocial e espiritual utilizada, pode-se manter o controle dos sintomas e de outros problemas psíquicos, sociais, culturais e espirituais. A realização do cuidado domiciliar permite a manutenção do paciente em casa com conforto e segurança, envolvendo o cuidado da família e do cuidador do paciente, dando-lhe suporte para garantir o controle dos sintomas e a dignidade no processo de morte. Além disso, existe a possibilidade de continuidade do cuidado da família mesmo após o falecimento, ajudando-a no enfrentamento do luto.

Tais atributos permitem à equipe de saúde da família coordenar o cuidado do paciente em $\mathrm{CP}$, garantindo acesso, orientando e permanecendo próxima, além de trazer mais segurança durante o processo de doença, $o$ que proporciona melhoria da qualidade de vida.

A ESF, hoje em expansão, apresenta, por seus atributos, maior capacidade para sustentar em abrangência e acompanhamento continuado essa população, sendo um ator em potencial para garantir cuidado à saúde.

\section{Referências}

1. Krause LH. Ainda que não se possa curar, sempre é possível cuidar. Revista HUPE. 2012;11:18-25.

2. Silva ML. O papel do profissional da Atenção Primária à Saúde em cuidados paliativos. Revista Brasileira de Medicina de Família e Comunidade [Internet]. 2013 Nov2; [Citado em 2015 Mai 30];9(30):45-53. Disponível em:http://www.rbmfc.org.br/ rbmfc/article/view/718

3. Queiroz AHAB, Pontes RJS, Souza AMA, et al. Percepção de familiares e profissionais de saúde sobre os cuidados no final da vida no âmbito da atenção primária à saúde. Ciênc. saúde coletiva[Internet]. 2013 Sep [cited 2015 May 30] ;18(9): 26152623. Disponível em: http://www.scielo.br/scielo.php?script=sci_arttext\&pid=S1413-81232013000900016\&lng=en. http:// dx.doi.org/10.1590/S1413-81232013000900016

4. Silva C, Cota L, Vieira R, et al. Doenças terminais, conhecimento essencial para o profissional da saúde. Psicologia Argumento. 2013 Jan./Mar;31(72):137-144.

5. Mendes JA, Lustosa MA, Andrade MCM. Paciente terminal, família e equipe de saúde. Rev. SBPH[periódico na Internet]. 2009 Jun [citado 2015 Maio30](1):151-173.Disponível em:http://pepsic.bvsalud.org/scielo.php?script=sci_arttext\&pid=S1516-08582009000100011\&lng=pt

6. Combinato DS, Queiroz M. Morte: uma visão psicossocial. Estudos de Psicologia. 2006;11(2):209-16.

7. Conselho Federal de Medicina. Código de ética médica: resolução CFM no 1.931, de 17 de setembro de 2009 (versão de bolso). Brasília: Conselho Federal de Medicina, 2010. Disponível em: https://portal.cfm.org.br/images/stories/biblioteca/ codigo\%20de\%20etica\%20medica.pdf

8. Lepargneur H. Bioética da eutanásia: argumentos éticos em torno da eutanásia. Bioética. 1999;7(1):41-48.

9. Temporão JG. Portaria № 741, De 19 de Dezembro de 2005. Disponível em:http://bvsms.saude.gov.br/bvs/saudelegis/ sas/2005/prt0741_19_12_2005.html

10. Matsumoto, DY. Cuidados paliativos: conceito, fundamentos e princípios. In: Carvalho RT, Parsons HA. Manual de cuidados paliativos ANCP. $2^{a}$ Edição. Porto Alegre: Sulina; 2012. p. 23-30.

11. Floriani C, Schramm F. Cuidados Paliativos: Interfaces, conflitos e necessidades. Ciencia e Saúde Coletiva. 2008; 13(SUP2):2123-32.

12. Floriani CA, Schramm FR. Desafios morais e operacionais da inclusão dos cuidados paliativos na rede de atenção básica. Cad. Saúde Pública[Internet]. 2007Sep [citado 2015 May 30]; 23( 9 ): 2072-2080. Disponível em:http://www.scielo.br/scielo. php?script=sci_arttext\&pid=S0102-311X2007000900015\&l$\mathrm{ng}=\mathrm{en}$

13. Fundação do Câncer. [homepage na Internet]. [Acesso em 25/05/2015]. Disponível em:http://cancer.org.br/a-fundacao/oque-fazemos/cuidados-paliativos/

14. Combinato DS, Martins STF. (Em defesa dos) Cuidados Paliativos na Atenção Primária à Saúde. O Mundo da Saúde. 2012;36(3):433-41.

15. Takeda S. A organização de serviços de Atenção Primária à Saúde. Duncan, B.B. Medicina ambulatorial: condutas de atenção primária baseadas em evidências. 4. ed. Porto Alegre: Artmed; 2013. p19-32.

16. Ministério da Saúde. Núcleo de Apoio à Saúde da Família (Cadernos de Atenção Básica, n. 39). Brasília: Ministério da Saúde, 2014. 116 p: Disponível em: http://bvsms.saude.gov.br/ bvs/publicacoes/nucleo_apoio_saude_familia_cab39.pdf

17. Ministério da Saúde. Política Nacional de Atenção Básica. Brasília : Ministério da Saúde, 2012. Disponível em : http://dab. saude.gov.br/portaldab/pnab.php 
Lilian Hennemann-Krause e cols. • Cuidados paliativos e medicina de família e comunidade: conceitos e interseções

18. Roncolleta A. Impacto das novas competências do médico de família: coordenação de cuidados no hospital e gerenciamento de pacientes crônicos no domicílio. O Mundo da saúde. 2009;33(1):108-13.

19. Koseki MN. Descentralização do tratamento a pacientes com câncer avançado sem possibilidade de cura. Campinas. SP:[s.n.], 2002.

20. Reigada C, Ribeiro J, Novellas, et al. O suporte à família em Cuidados Paliativos. Testos e Contextos. 2014 Jan./ Jun;13(1):159-69. 